\title{
Sampling Rate of Spatial Stochastic Processes with Independent Components in Modeling Random Search Paths
}

\author{
REIICHIRO KAWAI*
}

\begin{abstract}
Continuous-time modeling of random searches is designed to be robust to the sampling rate while the spatial model is required to be of rotation-invariant type, which is often computationally prohibitive. Such computational difficulty may be circumvented by employing a model with independent components. We demonstrate that its disadvantages in statistical properties are blurred under lower frequency. We propose a quantitative criterion for choice of the sampling rate at which a spatial model with independent components resembles a rotation-invariant model. Our findings have the potential to assist the observer to employ simpler models in the continuous-time framework to avoid expensive computation required for statistical inference.

PACS numbers: 87.23.-n, 87.10.Mn, 87.10.Ca
\end{abstract}

\section{Introduction}

Mathematical modeling of random searches is of great relevance in the field of ecology [1, 2, 3]. A large number of existing studies record the movement at equidistant time intervals and model such ordered sets of step length and turning angles directly with discrete-time random walks [4, 5]. Given the increasing availability of high resolution observation data, however, statistical inference for such time series data at different sampling rates has raised an important issue of robustness to the sampling rate, as estimation results for the discrete observation data depend largely on the sampling rate [6, 7]. In turn, continuous-time modeling [8] is designed to model the entire trajectory $\left\{X_{t}: t \in\left[0, t_{n, n}\right]\right\}$ based on the limited discrete observation $\left(X_{t_{n, 1}}, X_{t_{n, 2}}, \ldots, X_{t_{n, n}}\right)$ at equidistant time points $t_{n, k}:=k \Delta_{n}$, rather than fitting some random walk model, such as Lévy walks and correlated random walks. This continuous-time framework is thus purely robust to the sampling rate, while the discrete-time

*Published in Physical Review E (2012) 85(2) 021907. Email Address: reiichiro.kawai@le.ac.uk. Postal Address: Department of Mathematics, University of Leicester, Leicester LE1 7RH, UK. random walk framework does not allow for the concept of super-sampling, for instance. Given the increasing availability of high resolution data, it is of great interest to investigate more observations $(n \uparrow+\infty)$ at a higher sampling frequency $\left(\Delta_{n} \downarrow 0\right)$. The high frequency sampling reflects the best possible experiment environment in reality. Strictly speaking, observation over a whole interval is never possible even with recent high technology.

The main objective of this article is to suggest a possible simplification to modeling and analysis of random search patterns. To this end, we demonstrate that a simple motion (composed by two independent walks) resembles a more detailed model (a rotationally invariant process), when it is observed at low sampling frequencies. This fact also reinforces the idea [6, 7] that the temporal sampling of a given process influences the inference that one can make about it.

Let us begin with the most classical and tractable model; the Brownian motion. In Figure 1, we draw typical sample paths of the two-dimensional Brownian motion $\left\{t \gamma+B_{t}: t \geq 0\right\}$, where $\gamma:=\left[\gamma_{1}, \gamma_{2}\right]^{\top}$ indicates the deterministic drift, or equivalently the bias in movements, and where $\left\{B_{t}: t \geq 0\right\}$ is a centered two-dimensional Brownian motion with covariance matrix $\left[\begin{array}{cc}\sigma_{1}^{2} & \rho \sigma_{1} \sigma_{2} \\ \rho \sigma_{1} \sigma_{2} & \sigma_{2}^{2}\end{array}\right]$, with $\sigma_{1}>0, \sigma_{2}>0$ and $\rho \in[-1,+1]$. The value $\rho=0$ induces the Brownian motion with independent components, which is indeed rotationally invariant and thus best describes no directional preference among various values of $\rho \in[-1,+1]$. All the resulting computations are thus purely one-dimensional.
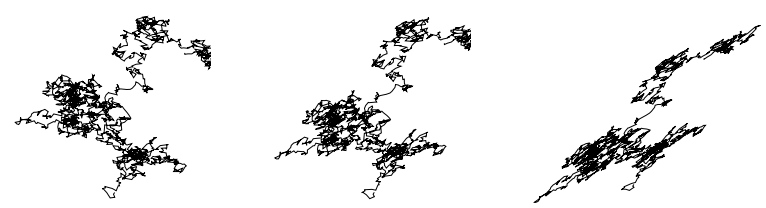

Figure 1: Typical sample paths of a two-dimensional Brownian motion with $\gamma=0, \sigma_{1}=\sigma_{2}$ and with $\rho=0$ (left), $\rho=0.4$ (center), and $\rho=0.8$ (right). 


\section{Spatio-Temporal Modeling}

There has been a widely accepted consensus [9] that the Brownian motion is not appropriate for modeling movement paths, as its sample path returns so frequently to the same place, which is unrealistic for searching for sparsely distributed resources. A first alternative to the Brownian motion is the stable Lévy motion model [10]. Its univariate motion has been used in several other fields of applications, such as statistical physics, queueing theory, and mathematical finance. In this article, we focus on a (nonGaussian) two-dimensional symmetric stable (Lévy) motion $\left\{X_{t}: t \geq 0\right\}$, which can be defined through the characteristic function [11, 12]

$$
\mathbb{E}\left[e^{i\left\langle y, X_{t}\right\rangle}\right]=\exp \left[t\left(i\langle y, \gamma\rangle+\int_{S}|\langle y, \xi\rangle|^{\alpha} \lambda(d \xi)\right)\right],
$$

where $\gamma \in \mathbb{R}^{2}$ indicates the bias in movements, where $\alpha \in$ $(0,2)$ is the stability index, and where $\lambda(d \xi)$ is a finite positive symmetric measure on $S$ (the unit sphere in $\mathbb{R}^{2}$ ), which governs the direction of jumps. The limit $\alpha \downarrow 0$ leads to ballistic motion. This stable motion, with $\gamma=0$, that is, no bias in movements, enjoys the self-similarity [11, 12, 13, 14, 15]; for any $h>0$,

$$
\left\{h^{-1 / \alpha} X_{h t}: t \geq 0\right\} \stackrel{\mathscr{L}}{=}\left\{X_{t}: t \geq 0\right\},
$$

which implies that the resultant patterns are fractal and no scale is characteristic. Hence, a self-similar set of points of fractal dimension $\alpha$ is visited by the walker. Unlike the Brownian motion, discrete observations of a stable motion is not identical to Lévy walks, that is, a discrete-time random walk with independent power-law distributed step lengths [7].

The two-dimensional symmetric stable Lévy motion (1) fails to capture all the statistical features of observed sample paths, while it turns out to be sufficiently useful for demonstration of the effect of sampling rate. As the univariate stable motion is thoroughly studied [11, 12] and the multivariate motion is still tractable in some instances, our discussion does not require unnecessarily intricate derivations but the basic known facts. In order to avoid overloading the article with nonessential mathematical details, we do not aim at ultimately realistic models in this article.

\subsection{Rotational Invariance}

We hereafter call rotation-invariant the case where the control measure $\lambda(d \xi)$ in (1) is moreover uniform on the unit sphere $S$; every independent jump has no preference in direction. In this setting, the characteristic function (1) reduces to

$$
\mathbb{E}\left[e^{i\left\langle y, X_{t}\right\rangle}\right]=e^{t\left(i\langle y, \gamma\rangle-2 \sigma^{\alpha}\|y\|^{\alpha}\right)},
$$

with some $\sigma>0$. Its sample path on a finite horizon $[0, T]$ can be generated through the series representation [11]

$$
\left\{t \gamma+C_{\alpha, \sigma, T}^{-1 / \alpha} \sum_{n=1}^{+\infty} \Gamma_{n}^{-1 / \alpha} \xi_{n} \mathbb{1}\left(T_{n} \leq t\right): t \in[0, T]\right\},
$$

where $\left\{T_{n}\right\}_{n \in \mathbb{N}}$ is a sequence of independent and identically distributed (iid) uniform random variables on $[0, T]$, $\left\{\Gamma_{n}\right\}_{n \in \mathbb{N}}$ are arrival times of a standard Poisson process, $\left\{\eta_{n}\right\}_{n \in \mathbb{N}}$ is a sequence of iid uniform random variables on $[0,2 \pi), \xi_{n}=\left[\cos \eta_{n}, \sin \eta_{n}\right]^{\top}$ for each $n \in \mathbb{N}$, and $C_{\alpha, \sigma, T}:=\Gamma(1-\alpha) \cos (\pi \alpha / 2) /\left(2 \sigma^{\alpha} T\right)$. In Figure 2, we draw typical sample paths of a rotation invariant stable motion in $\mathbb{R}^{2}$ on the unit time interval $[0,1]$ under three different sampling frequencies.
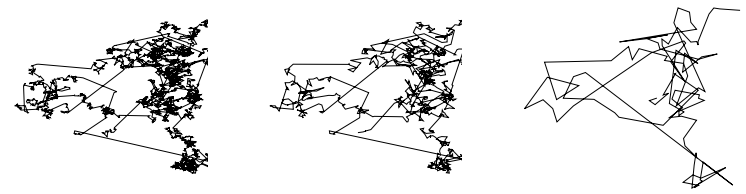

Figure 2: Typical sample paths of a two-dimensional rotationinvariant stable motion $(\gamma, \alpha, \sigma)=(0.0,1.5,1.0)$ on the unit time interval $[0,1]$ with three different sampling frequencies; 5000 increments (leftmost), 1000 increments (center), and 100 increments (rightmost).

Despite its rich description, such rotation-invariant spatio-temporal models is often out of our reach from a computational point of view. For instance, maximum likelihood estimation is far from tractable as the probability density function of its increments is purely twodimensional and is not available in closed form.

\subsection{Independent Components}

Such practical issues may be circumvented by employing the stable motion with independent components, as it consists of two independent one-dimensional stable motions. In particular, statistical methods are well-studied [16] for the one-dimensional setting. Let the control measure $\lambda(d \xi)$ in (1) be a sum of four Dirac delta measures concentrated at $[1,0]^{\top},[0,1]^{\top},[-1,0]^{\top}$ and $[0,-1]^{\top}$, up to a constant. The characteristic function (1) reduces to

$$
\mathbb{E}\left[e^{i\left\langle y, X_{t}\right\rangle}\right]=e^{t\left(i y_{1} \gamma_{1}-\sigma^{\alpha}\left|y_{1}\right|^{\alpha}\right)} e^{t\left(i y_{2} \gamma_{2}-\sigma^{\alpha}\left|y_{2}\right|^{\alpha}\right)},
$$


with $y=\left[y_{1}, y_{2}\right]^{\top}$ and some $\sigma>0$, indicating independence of the two components. Each component has the so-called inverse power-law tail; a random variable $Z$ with $\mathbb{E}\left[e^{i y Z}\right]=\exp \left[-\sigma^{\alpha}|y|^{\alpha}\right]$ has the probability tail behavior $[11,12]$

$$
\mathbb{P}(Z>x) \sim \frac{\sigma^{\alpha}}{2 \Gamma(1-\alpha) \cos (\pi \alpha / 2)} x^{-\alpha}, \quad x \uparrow+\infty,
$$

where " $"$ above indicates asymptotic equivalence. The spatial stable motion with independent components can also be simulated through an infinite shot noise series representation (3), where $\left\{\eta_{n}\right\}_{n \in \mathbb{N}}$ here is a sequence of iid uniform random variables on $\{0, \pi / 2, \pi, 3 \pi / 2\}$ instead. We draw in Figure 3 typical sample paths of a twodimensional stable motion with independent components on the unit time interval $[0,1]$ with three different sampling frequencies. Under high frequency sampling, the trajectory appears to move too orthogonally to correctly describe movement paths. If the sampling frequency is relatively low, however, the undesired orthogonal looks tend to disappear, as the movement of each component gets more averaged and gives a sufficient impression of rotation-invariance. Note that the stable motion with independent increments still satisfies (2) and is thus free of scale, while Figure 3 does not account for the fractal scaling (2), as

$$
\begin{aligned}
\left(X_{1 \Delta_{n_{1}}}, \ldots, X_{n_{1} \Delta_{n_{1}}}\right) & \stackrel{\mathscr{L}}{=}\left(n_{1} / n_{3}\right)^{-1 / \alpha}\left(X_{1 \Delta_{n_{3}}}, \ldots, X_{n_{1} \Delta_{n_{3}}}\right) \\
& \stackrel{\mathscr{L}}{\neq}\left(X_{1 \Delta_{n_{3}}}, \ldots, X_{n_{3} \Delta_{n_{3}}}\right)
\end{aligned}
$$

where $\left(X_{1 \Delta_{n_{1}}}, \ldots, X_{n_{1} \Delta_{n_{1}}}\right)$ and $\left(X_{1 \Delta_{n_{3}}}, \ldots, X_{n_{3} \Delta_{n_{3}}}\right)$ correspond respectively to its leftmost and rightmost figures, with $n_{1}=5000, n_{3}=100$ and $\Delta_{n}:=1 / n$.

\subsection{Asymptotic Isotropicity}

The observations made in Figure 1 and 3 can be justified as follows. Let $\phi_{\alpha}\left(x ; \sigma, \Delta_{n}\right)$ be the probability density function of the centered symmetric stable distribution on $\mathbb{R}$ whose characteristic function is given by $e^{-\Delta_{n} \alpha^{-1} \sigma^{\alpha}|z|^{\alpha}}$, that is,

$$
\phi_{\alpha}\left(x ; \sigma, \Delta_{n}\right):=\frac{1}{2 \pi} \int_{\mathbb{R}} e^{-i x z} e^{-\Delta_{n} \alpha^{-1} \sigma^{\alpha}|z|^{\alpha}} d z, \quad x \in \mathbb{R},
$$

with the scale $\sigma>0$ and the stepsize $\Delta_{n}>0$. (Note that this formulation covers the Gaussian case $\alpha=2$.) The probability density function $\phi_{\alpha}\left(x, y ; \sigma, \Delta_{n}\right)$ of the twodimensional stable law with independent components, each of which admits the probability density function $\phi_{\alpha}\left(x ; \sigma, \Delta_{n}\right)$, is given by

$\phi_{\alpha}\left(x, y ; \sigma, \Delta_{n}\right)=\phi_{\alpha}\left(x ; \sigma, \Delta_{n}\right) \phi_{\alpha}\left(y ; \sigma, \Delta_{n}\right), \quad[x, y]^{\top} \in \mathbb{R}^{2}$, due to independence of the two components. Let $(x, y)=$ $(\psi \cos \theta, \psi \sin \theta)$ with some $\psi>0$ and $\theta \in[0,2 \pi)$. In the Gaussian case $\alpha=2$, it is straightforward that

$$
\phi_{2}\left(x, y ; \sigma, \Delta_{n}\right)=\frac{1}{2 \pi \Delta_{n} \sigma^{2}} e^{-\frac{x^{2}+y^{2}}{2 \Delta_{n} \sigma^{2}}}=\frac{1}{2 \pi \Delta_{n} \sigma^{2}} e^{-\frac{\psi^{2}}{2 \Delta_{n} \sigma^{2}}} .
$$

Independence of the absolute turning angle $\theta$, regardless of $\left(\sigma, \Delta_{n}\right)$, proves that the Gaussian law with independent components is indeed rotationally invariant. In turn, it holds that for $\alpha \in(0,2)$ and each $(x, y)$,

$$
\begin{aligned}
\phi_{\alpha}\left(x, y ; \sigma, \Delta_{n}\right) & =\frac{1}{2 \pi} \int_{\mathbb{R}} e^{-\sigma^{\alpha}\left|z_{1}\right|^{\alpha}} \cos \left(x \Delta_{n}^{-1 / \alpha} z_{1}\right) \frac{d z_{1}}{\Delta_{n}^{1 / \alpha}} \\
& \frac{1}{2 \pi} \int_{\mathbb{R}} e^{-\sigma^{\alpha}\left|z_{2}\right|^{\alpha}} \cos \left(y \Delta_{n}^{-1 / \alpha} z_{2}\right) \frac{d z_{2}}{\Delta_{n}^{1 / \alpha}} \\
& \sim\left(\frac{1}{2 \pi \Delta_{n}^{1 / \alpha}} \int_{\mathbb{R}} e^{-\sigma^{\alpha}|z|^{\alpha}} d z\right)^{2} \\
& =\left(\frac{\Gamma(1 / \alpha)}{\pi \Delta_{n}^{1 / \alpha} \sigma \alpha}\right)^{2}
\end{aligned}
$$

for a large stepsize $\Delta_{n}$, where the equivalence (5) holds by $\cos \left(x \Delta_{n}^{-1 / \alpha} z\right)=1+O\left(x^{2} \Delta_{n}^{-2 / \alpha} z^{2}\right)$ as $\Delta_{n} \uparrow+\infty$. In particular, in the case of the Cauchy Lévy motion $(\alpha=1)$ for which probability density function is available in closed form, it holds that

$$
\begin{aligned}
\phi_{1}\left(x, y ; \sigma, \Delta_{n}\right) & =\frac{\left(\Delta_{n} \sigma\right)^{2}}{\pi^{2}\left(x^{2}+\left(\Delta_{n} \sigma\right)^{2}\right)\left(y^{2}+\left(\Delta_{n} \sigma\right)^{2}\right)} \\
& \sim \frac{1}{\left(\pi \Delta_{n} \sigma\right)^{2}} .
\end{aligned}
$$

This fact indicates asymptotic independence of the density function from the turning angle $\theta$ at a relatively low sampling rate. This accounts for the phenomena of Figure 3 , that is, the stable law on $\mathbb{R}^{2}$ with independent components looks rotationally invariant, if the stepsize $\Delta_{n}$ is relatively large.

So far, we have assumed, without justification, that there exists no bias in movements $\gamma=0$ in the model (1) and the series representation (3). In fact, such unbiasedness is not a crucial assumption, while a bias is not essential in our discussion either. We draw in Figure 4 typical sample paths of a two-dimensional stable motion with the bias $\gamma \neq 0$. The movement in the left figure is the rotationally invariant movement under high frequency sampling in the leftmost of Figure 2, together with the artificial bias $\gamma=[+1.0,-1.0]^{\top}$. The right figure indicates the movement with independent components in the leftmost of Figure 3 together with the same bias. Obviously, the addition of bias changes the overall tendency of movements; In this case, by the bias $\gamma=[+1.0,-1.0]^{\top}$, the sample paths are 

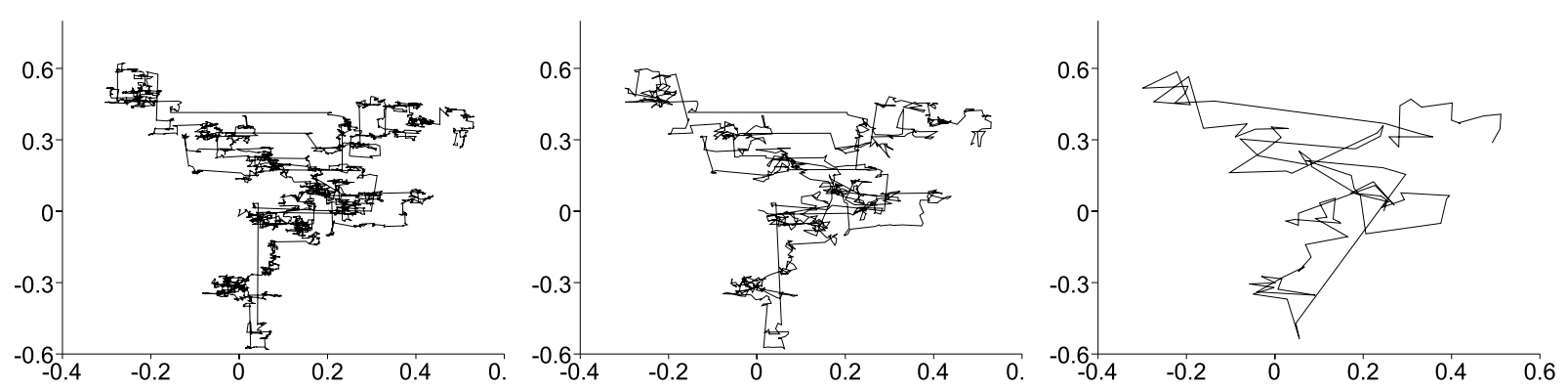

Figure 3: Typical sample paths of a two-dimensional stable motion with independent components $(\gamma, \alpha, \sigma)=$ $(0.0,1.5,1.0)$ on the unit time interval $[0,1]$ with three different sampling frequencies; 5000 increments (leftmost), 1000 increments (center), and 100 increments (rightmost).

dragged gradually in the right-below direction. As can be observed, however, neither "the obvious difference between rotation invariance and independent components" nor "the orthogonal-looking" is affected by the bias in the high frequency sampling framework. The reason is quite simple. In the case of heavy-tailed movements, such as the stable motion and the Pareto random walk, the sample paths tend to be dominated by occasional unusually large jumps, which cannot be much influenced by small gradual biases. In turn, in the low frequency sampling framework, since a stable motion with independent components without bias resembles a rotationally invariant process without bias, the addition of bias does not affect the resemblance between those two. We thus assume no bias in movements $\gamma=0$ hereafter.
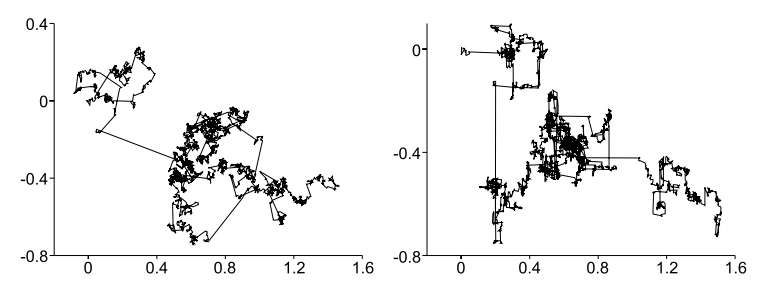

Figure 4: Typical sample paths of a two-dimensional stable motion on the unit time interval $[0,1]$, with $(\alpha, \sigma)=(1.5,1.0)$ and a strong bias $\gamma=[+1.0,-1.0]^{\top}$. Rotationaly invariant (left) and independent components (right).

\section{Methods and Results}

The rigorous criterion for independent components to look rotationally invariant is evidently a uniform angular distribution of discrete steps, which is realized exactly when $\alpha=2$ irrespective of the sampling frequency, as seen in (4). In the case of non-Gaussian $(\alpha \neq 2)$, however, the asymptotic independence (5) implies that such uniformity of the angular distribution is impossible with a finite stepsize $\Delta_{n}$. Instead, we propose an alternative quantitative way as to choice of the sampling rate in such a way that the stable motion with independent components may look rotationally invariant. Let $\left\{Z_{t}: t \geq 0\right\}$ be a twodimensional stable motion with independent components, and let $Z_{t}=:\left[X_{t}, Y_{t}\right]^{\top}$ where $\left\{X_{t}: t \geq 0\right\}$ and $\left\{Y_{t}: t \geq 0\right\}$ are iid centered symmetric one-dimensional stable motions. It is well known that the probability tails behave like [11, 12]

$$
\mathbb{P}\left(\left|X_{\Delta_{n}}\right|>x_{h}\right) \sim \frac{\Delta_{n} \sigma^{\alpha} x_{h}^{-\alpha}}{\Gamma(1-\alpha) \cos (\pi \alpha / 2)}, \quad x_{h} \uparrow+\infty .
$$

In the meantime, due to symmetry of the law about the origin, the small deviation is given by

$$
\mathbb{P}\left(\left|X_{\Delta_{n}}\right| \leq x_{l}\right) \sim 2 x_{l} \frac{\Gamma(1 / \alpha)}{\Delta_{n}^{1 / \alpha} \sigma \pi \alpha}, \quad x_{l} \downarrow 0,
$$

which is the product of the width $2 x_{l}$ and the height, that is the probability density function of $X_{\Delta_{n}}$ at the origin, explicitly computable as $(2 \pi)^{-1} \int_{\mathbb{R}} e^{-i y 0-\Delta_{n} \sigma^{\alpha}|y|^{\alpha}} d y$. The undesirable event is, in principle, that one component moves a lot, while the other barely moves over some observation interval $\left[t, t+\Delta_{n}\right]$, since then such an increment looks nearly orthogonal in $\mathbb{R}^{2}$. In the other words, it would be preferable to keep the probability of orthogonallooking increments as small as possible over a sample path. Such orthogonal-looking increments can be expressed as

$$
\left\{\left|X_{\Delta_{n}}\right| \leq x_{l},\left|Y_{\Delta_{n}}\right|>x_{h}\right\} \cup\left\{\left|X_{\Delta_{n}}\right|>x_{h},\left|Y_{\Delta_{n}}\right| \leq x_{l}\right\},
$$

where $\arctan \left(x_{h} / x_{l}\right) \approx \pi / 2$. Suppose, for a moment, that the thresholds $x_{h}$ and $x_{l}$ are fixed very large and very small, respectively. The probability of our interest is then 
given by

$$
\begin{aligned}
& \mathbb{P}\left(\left\{\left|X_{\Delta_{n}}\right| \leq x_{l},\left|Y_{\Delta_{n}}\right|>x_{h}\right\} \cup\left\{\left|X_{\Delta_{n}}\right|>x_{h},\left|Y_{\Delta_{n}}\right| \leq x_{l}\right\}\right) \\
& \quad=2 \mathbb{P}\left(\left\{\left|X_{\Delta_{n}}\right| \leq x_{l},\left|Y_{\Delta_{n}}\right|>x_{h}\right\}\right) \\
& \quad=2 \mathbb{P}\left(\left|X_{\Delta_{n}}\right| \leq x_{l}\right) \mathbb{P}\left(\left|Y_{\Delta_{n}}\right|>x_{h}\right) \\
& \quad \sim 1 \wedge \frac{4 \sigma^{\alpha-1} \Gamma(1 / \alpha) x_{h}^{-\alpha} x_{l}}{\pi \alpha \Gamma(1-\alpha) \cos (\pi \alpha / 2)} \Delta_{n}^{1-1 / \alpha}=: p_{n},
\end{aligned}
$$

where the equalities holds by independence and stationarity of components and of the stable motion and where the last asymptotics holds by (6) and (7). Finally, by definition, a single sample path is observed at $n$ equidistant points in time, thus the probability of $k$ orthogonallike increments over a single sample path is given by ${ }_{n} C_{k} p_{n}^{k}\left(1-p_{n}\right)^{n-k}$. We look at the quantity

$$
\sum_{k=K+1}^{n}{ }_{n} C_{k} p_{n}^{k}\left(1-p_{n}\right)^{n-k}=: P_{n, K}\left(\alpha, \sigma, x_{h}, x_{l}\right),
$$

indicating the probability of more than $K$ orthogonallooking large increments over a single sample path, which is very easy to compute. Hereafter, we say that a sample path with $K$ or more such increments resembles a stable motion with independent components.

The remaining important factor in our quantitative analysis is choice of the thresholds $x_{h}$ and $x_{l}$. Although they are not required to be constant as the ratio $x_{h} / x_{l}$ only matters in the definition (8) of "orthogonal-looking increments", we focus on the setting with prescribed constants $x_{h}$ and $x_{l}$ since (i) from a practical point of view, just as seen in Figure 3, the overall scale is irrelevant to the sampling stepsize $\Delta_{n}$; (ii) from a theoretical point of view, this validates the use of the deviations (6) and (7). An important point is that a bunch of very orthogonal, but tiny, jumps do not affect much of the visual looking. In the specific case of Figure 3, a few (for example, five) orthogonal-looking jumps with length longer than 0.4 seem to make the sample path look truly orthogonal. Here, we test $x_{h}=0.2$ and $x_{h}=0.4$ with $x_{l}=x_{h} / \tan \theta$, with $\theta=80^{\circ}, 82^{\circ}, 84^{\circ}, 86^{\circ}$. With the aid of graphs like in Figure 5] one may roughly choose an appropriate observation number $n$ (and consequently, the observation stepsize $\Delta_{n}(=T / n)$ ). In short, with smaller thresholds $x_{h}$ and $x_{l}$ or with more orthogonal-looking movements allowed, the observer may look at the higher frequency of a model with independent components.

\section{Discussion}

We have demonstrated that a spatio-temporal model with independent components may look rotationally invariant when the sampling rate is not very high and have proposed
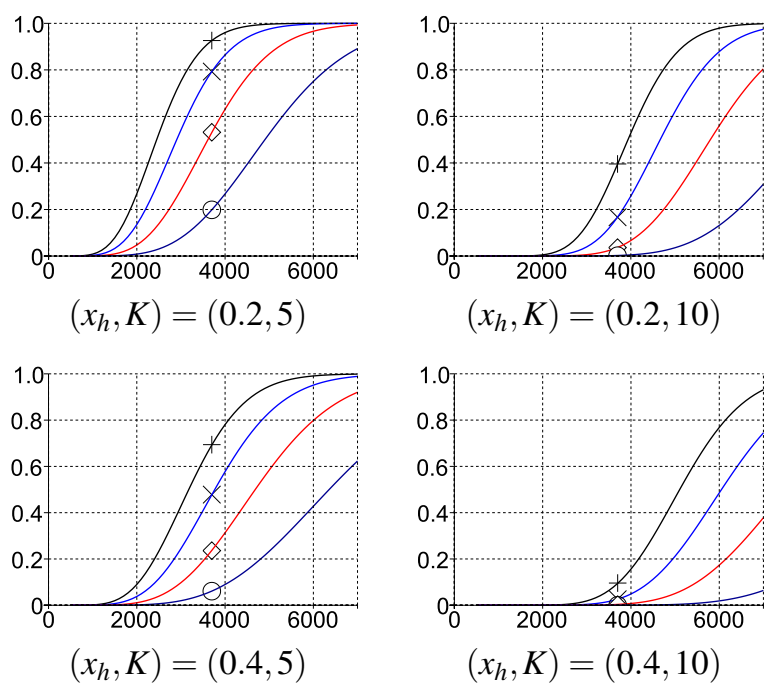

Figure 5: Plots of $P_{n, K}\left(1.5,1, x_{h}, x_{l}\right)$ against $n$, with $x_{l}=$ $x_{h} / \tan \theta$. The lines with "+", " $\times "$, , $\diamond "$ and "॰" correspond, respectively, to $\theta=80^{\circ}, 82^{\circ}, 84^{\circ}, 86^{\circ}$.

a quantitative criterion for choice of the sampling rate at which this phenomenon takes place in the stable motion model. In practice, spatio-temporal models with independent components are mathematically more tractable as it consists of two independent one-dimensional motions, and may thus be employed so as to avoid prohibitive computation under low frequency sampling. Moreover, in lattice environments [17], such motions are useful by nature for modeling random searches even in the high frequency observation framework.

There are further statistical properties to be taken into account for better modeling, such as aggregational Gaussianity [8], autocorrelation in step length [6, 19], and memory in direction [18]. In particular, non-Markovian properties (such as the latter two) tend to be less observable when movement paths are observed in less detail, that is, under low frequency sampling. For example, as is well known [9, 20], a correlated random walk [18] resembles Brownian motion at a low sampling rate, due to the tangling impact of the turning angles. At a relatively low observation rate where such non-Markovian properties become negligible, the stable Lévy motion is still useful for modeling random search paths.

The phenomenon "a distant observation veils the pattern" can be seen in various fields of application in which our approach has been applied in a similar manner. For example, the raster format is an image digitization technique based upon this phenomenon. A raster image is made up by pixels of an equal spatial size (in our context, temporal stepsize $\Delta_{n}$ ) with different colors, and 
is essentially indistinguishable from its original image (rotationally invariant movements) through a distant observation (large $\Delta_{n}$ ), while a lattice pattern (orthogonal movements with independent components) will show up through a very close look $\left(\Delta_{n} \downarrow 0\right)$. The raster technique allows us to store an analog image as digital data with some loss of accuracy, corresponding to lighter computational requirements thanks to decomposition of a purely two-dimensional movement into two independent onedimensional movements.

The criterion of Section 3 may be further strengthened with statistical testing techniques. As far as the twodimensional Brownian motion (or equivalently the twodimensional normal random vectors) is concerned, for example, the well known $t$-test of Gaussian independence [21], whether " $\rho=0$ " or " $\rho \neq 0$ ", may be useful for our purpose. For the two-dimensional stable law, such useful techniques does not seem to exist, as the variance (and thus the correlation) of the stable distribution is infinite.

Our findings reconfirm importance of super-sampling and a priori extra conceptual information of the trajectory; without high frequency sampling, the observer may overlook a hidden essential pattern. For instance, without a priori knowledge, a human walking trajectory in a lattice-like city (perhaps through satellite observation) could be interpreted mistakenly as a movement with no directional preference, when sampling frequency is too low. In a boarder sense, our study is expected to facilitate further investigations of timely issues in random search modeling, such as statistical inference and experimental settings for complex movements, for instance, with intermittency.

\section{Acknowledgement}

The author would like to thank Sergei Petrovskii for stimulating discussions and the referees for various valuable comments and suggestions.

\section{References}

[1] Bartumeus, F., Catalan, J., Journal of Physics A: Mathematical and Theoretical, 42, 434002 (2009)

[2] Codling, E.A., Plank, M.J., Benhamou, S., J. R. Soc. Interface, 5, 813-834 (2008)

[3] Turchin, P., Quantitative Analysis of Movement: Measuring and Modeling Population Redistribution in Animals and Plants, Sunderland, MA: Sinauer. (1988)

[4] Edwards, A.M. et al., Nature, 499, 1044-8 (2007)

[5] Humphries, N.E., et al., Nature, 465, 1066-1069 (2010)

[6] Kawai, R., Petrovskii, S., Multiscale properties of random walk models of animal movement: lessons from statistical inference, Proceedings of the Royal Society A, doi:10.1098/rspa.2011.0665.
[7] Plank, M.J,, Codling, E.A., Ecology, 90(12) 3546-3553 (2009)

[8] Kawai, R., Continuous-time modeling of random searches: statistical properties and inference, under review.

[9] Viswanathan, G.M., Nature, 465, 1018-1019 (2010)

[10] Shlesinger, M.F., Zaslavsky, G.M., Frisch, U. (eds) Lévy Flights and Related Topics in Physics, Springer, Berlin. (1995)

[11] Samorodnitsky, G., Taqqu, M.S., Stable non-Gaussian random processes, Chapman \& Hall, New York. (1994)

[12] Sato, K., Lévy processes and infinitely divisible distributions, Cambridge University Press. (1999)

[13] Plank, M.J., James, A., Journal of the Royal Society Interface, 5, 1077-86 (2008)

[14] Reynolds, A.M., et al., Ecology, 88(8) 1955-1961 (2007)

[15] Sims, D. et al., Nature, 451, 1098-102 (2008)

[16] Nolan, J.P., In: Lévy Processes - Theory and Applications (ed. Barndorff-Nielsen, O.-E., Mikosch, T., Resnick, S.I.), 379-400, Birkhäuser, Boston. (2001)

[17] Santos, M.C., Viswanathan, G.M., Raposo, E.P., da Luz, M.G.E., Physical Review E, 77, 041101 (2008)

[18] Kareiva, P.M., Shigesada, N., Oecologia, 56 234-238 (1983)

[19] Reynolds, A.M., Journal of Physics A: Mathematical and Theoretical, 42, 434006 (2009)

[20] Raposo, E.P., Buldyrev, S.V., da Luz, M.G.E., Viswanathan, G.M., Stanley, H.E., Journal of Physics A: Mathematical and Theoretical, 42, 434003 (2009)

[21] Fisher, R.A., Biometrika, 10 507-521 (1915) 